Article

\title{
Quantum Current Algebra Symmetries and Integrable Many-Particle Schrödinger Type Quantum Hamiltonian Operators
}

\author{
Dominik Prorok ${ }^{1}$ and Anatolij Prykarpatski ${ }^{2, *}$ \\ 1 Department of Physics and Computer Science at AGH University of Science at Technology, \\ 31-155 Krakow, Poland \\ 2 The Institute of Mathematics at the Department of Physics, Mathematics and Computer Science of the \\ Cracov University of Technology, 31-155 Krakow, Poland \\ * Correspondence: pryk.anat@cybergal.com
}

Received: 23 June 2019; Accepted: 24 July 2019; Published: 1 August 2019

\begin{abstract}
Based on the G. Goldin's quantum current algebra symmetry representation theory, have succeeded in explaining a hidden relationship between the quantum many-particle Hamiltonian operators, defined in the Fock space, their factorized structure and integrability. Interesting for applications quantum oscillatory Hamiltonian operators are considered, the quantum symmetries of the integrable quantum Calogero-Sutherland model are analyzed in detail.
\end{abstract}

Keywords: fock space; current algebra representations; hamiltonian reconstruction; quantum integrability; quantum symmetries

PACS: 11.10.Ef; 11.15.Kc; 11.10.-z; 11.15.-q; 11.10.Wx; 05.30.-d

\section{Introduction}

In this work we developed investigations of local quantum current algebra symmetry representations, suggested and devised before by G. Goldin, in suitably renormalized Fock spaces and applying them to constructing the related finite-particle factorized representations for corresponding secondly-quantized many-particle integrable Hamiltonian operators. As examples we have studied in detail the factorized structure of Hamiltonian operators, describing such quantum integrable spatially many- and one-dimensional models as generalized oscillatory and Calogero-Sutherland dynamical systems of spin-less bose-particles.

\section{The Generalized Fock Type Spaces and Nonrelativistic Quantum Currents Algebra Structure}

\subsection{Preliminaries: Fock Type Hilbert Space, Nonrelativistic Quantum Current Algebra and Its Representations}

Let us consider the canonical Fock space [1-8], that is the direct sum

$$
\Phi=\oplus_{n \in \mathbb{Z}_{+}} \Phi_{(s)}^{\otimes n}
$$

where subspaces $\Phi_{(s)}^{\otimes n}, n \in \mathbb{Z}_{+}$, are the symmetrized tensor products of a Hilbert space $\mathcal{H} \simeq L_{2}^{(s)}\left(\mathbb{R}^{m} ; \mathbb{C}\right)$. If a vector $\varphi:=\left(\varphi_{0}, \varphi_{1}, \ldots, \varphi_{n}, \ldots\right) \in \Phi$, its norm

$$
\|\varphi\|_{\Phi}:=\left(\sum_{n \in \mathbb{Z}_{+}}\left\|\varphi_{n}\right\|_{n}^{2}\right)^{1 / 2}
$$


where $\varphi_{n} \in \Phi_{(s)}^{\otimes n} \simeq L_{2,(s)}\left(\left(\mathbb{R}^{m}\right)^{\otimes n} ; \mathbb{C}\right)$ and $\|\ldots\|_{n}$ is the corresponding norm in $\Phi_{(s)}^{\otimes n}$ for all $n \in \mathbb{Z}_{+}$. Note here that there holds the corresponding rigging [1,2] of the Hilbert spaces $\Phi_{(s)}^{\otimes n}, n \in \mathbb{Z}_{+}$, that is

$$
\mathcal{D}_{(s)}^{n} \subset \Phi_{(s),+}^{\otimes n} \subset \Phi_{(s)}^{\otimes n} \subset \Phi_{(s),-}^{\otimes n}
$$

with some suitably chosen dense and separable topological spaces of symmetric functions $\mathcal{D}_{(s)}^{n}, n \in \mathbb{Z}_{+}$, allowing to describe point wise particle objects in $\mathbb{R}^{m}$ by means of the corresponding generalized positive $\Phi_{(s),+}^{\otimes n}$ and negative Hilbert spaces $\Phi_{(s),-}^{\otimes n}$. Concerning expansion (1) one obtains by means of projective and inductive limits $[1,2,9,10]$ the quasi-nucleus rigging of the Fock space $\Phi$ exactly in the form (3).

Consider now any vector $\left.\mid(\alpha)_{n}\right) \in \Phi_{(s)}^{\otimes n}, n \in \mathbb{Z}_{+}$, which can be written [2,3,11-13] in the following canonical Dirac ket-form:

$$
\left.\left.\mid(\alpha)_{n}\right):=\mid \alpha_{1}, \alpha_{2}, \ldots, \alpha_{n}\right),
$$

where, by definition,

$$
\left.\left.\left.\left.\mid \alpha_{1}, \alpha_{2}, \ldots, \alpha_{n}\right):=\frac{1}{\sqrt{n !}} \sum_{\sigma \in S_{n}} \mid \alpha_{\sigma(1)}\right) \otimes \mid \alpha_{\sigma(2)}\right) \ldots \mid \alpha_{\sigma(n)}\right)
$$

and vectors $\left.\mid \alpha_{j}\right) \in \Phi_{(s)}^{\otimes 1}\left(\mathbb{R}^{m} ; \mathbb{C}\right) \simeq \mathcal{H}, j \in \mathbb{Z}_{+}$, are bi-orthogonal to each other, that is $\left(\alpha_{k} \mid \alpha_{j}\right)_{\mathcal{H}}=\delta_{k, j}$ for any $k, j \in \mathbb{Z}_{+}$. The corresponding scalar product of base vectors as (5) is given as follows:

$$
\begin{aligned}
& \left((\beta)_{n} \mid(\alpha)_{n}\right):=\left(\beta_{n}, \beta_{n-1}, \ldots, \beta_{2}, \beta_{1} \mid \alpha_{1}, \alpha_{2}, \ldots, \alpha_{n-1}, \alpha_{n}\right) \\
= & \sum_{\sigma \in S_{n}}\left(\beta_{1} \mid \alpha_{\sigma(1)}\right)_{\mathcal{H}} \ldots\left(\beta_{n} \mid \alpha_{\sigma(n)}\right)_{\mathcal{H}}:=\operatorname{per}\left\{\left(\beta_{i} \mid \alpha_{j}\right)_{\mathcal{H}}\right\}_{i, j=\overline{1, n}},
\end{aligned}
$$

where "per" denotes the permanent of matrix and $<\cdot \mid \cdot>$ is the corresponding scalar product in the Hilbert space $\mathcal{H}$. Based now on the representation (4) one can define an operator $a^{+}(\alpha): \Phi_{(s)}^{\otimes n} \longrightarrow \Phi_{(s)}^{\otimes(n+1)}$ for any $|\alpha\rangle \in \mathcal{H}$ as follows:

$$
\left.\left.a^{+}(\alpha) \mid \alpha_{1}, \alpha_{2}, \ldots, \alpha_{n}\right):=\mid \alpha, \alpha_{1}, \alpha_{2}, \ldots, \alpha_{n}\right),
$$

which is called the "creation" operator in the Fock space $\Phi$. The adjoint operator $a(\beta):=$ $\left(a^{+}(\beta)\right)^{*}: \Phi_{(s)}^{\otimes(n+1)} \longrightarrow \Phi_{(s)}^{\otimes n}$ with respect to the Fock space $\Phi$ (1) for any $|\beta\rangle \in \mathcal{H}$, called the "annihilation" operator, acts as follows:

$$
\left.\left.a(\beta) \mid \alpha_{1}, \alpha_{2}, \ldots, \alpha_{n+1}\right):=\sum_{\sigma \in S_{n}}\left(\beta \mid \alpha_{j}\right) \mid \alpha_{1}, \alpha_{2}, \ldots, \alpha_{j-1}, \hat{\alpha}_{j}, \alpha_{j+1}, \ldots, \alpha_{n+1}\right),
$$

where the "hat" over a vector denotes that it should be omitted from the sequence.

It is easy to check that the commutator relationship

$$
\left[a(\alpha), a^{+}(\beta)\right]=(\alpha \mid \beta)_{\mathcal{H}}
$$

holds for any vectors $\mid \alpha) \in \mathcal{H}$ and $\mid \beta) \in \mathcal{H}$. Expression (9), owing to the quasi-nucleusly [1,2] rigged Fock space (1), can be naturally extended to the general case, when vectors $\mid \alpha$ ) and $\mid \beta) \in \mathcal{H}_{-}$, where $\mathcal{H}_{-}$denotes the corresponding negative Hilbert space of generalized functions, conserving its usual form. In particular, taking $\mid \alpha):=\mid \alpha(x))=\frac{1}{\sqrt{2 \pi}} e^{i\langle\lambda \mid x\rangle} \in \mathcal{H}_{-}:=L_{2,-}\left(\mathbb{R}^{m} ; \mathbb{C}\right)$ for any $x \in \mathbb{R}^{m}$, one easily gets from (9) that

$$
\left[a(x), a^{+}(y)\right]=\delta(x-y),
$$

where we put, by definition, $\langle\cdot \mid \cdot\rangle$ the usual scalar product in the $m$-dimensional Euclidean space $\left(\mathbb{R}^{m} ;<\cdot \mid \cdot>\right), a^{+}(x):=a^{+}(\alpha(x))$ and $a(y):=a(\alpha(y))$ for all $x, y \in \mathbb{R}^{m}$ and denoted by $\delta(\cdot)$ the classical Dirac delta-function. 
The construction above makes it possible to observe easily that there exists the unique vacuum vector $\left.\mid \Omega_{0}\right) \in \mathcal{H}_{+}$, such that for any $x \in \mathbb{R}^{m}$

$$
\left.a(x) \mid \Omega_{0}\right)=0,
$$

and the set of vectors

$$
\left.\left(\prod_{j=1}^{n} a^{+}\left(x_{j}\right)\right) \mid \Omega_{0}\right) \in \Phi_{(s)}^{\otimes n}
$$

is total in $\Phi_{(s)}^{\otimes n}$, that is their linear integral hull over the functional spaces $\Phi_{(s)}^{\otimes n}$ is dense in the Hilbert space $\Phi_{(s)}^{\otimes n}$ for every $n \in \mathbb{Z}_{+}$. This means that for any vector $\varphi \in \Phi$ the following canonical representation

$$
\left.\varphi=\sum_{n \in \mathbb{Z}_{+}}^{\oplus} \frac{1}{\sqrt{n !}} \int_{\left(\mathbb{R}^{m}\right)^{n}} \varphi_{n}\left(x_{1}, \ldots, x_{n}\right) a^{+}\left(x_{1}\right) a^{+}\left(x_{2}\right) \ldots a^{+}\left(x_{n}\right) \mid \Omega_{0}\right)
$$

holds with the Fourier type coefficients $\varphi_{n} \in \Phi_{(s)}^{\otimes n}$ for all $n \in \mathbb{Z}_{+}$with $\Phi_{(s)}^{\otimes 1} \simeq \mathcal{H}=L_{2}^{(s)}\left(\mathbb{R}^{m} ; \mathbb{C}\right)$. The latter is naturally endowed with the Gelfand type quasi-nucleus rigging, dual to

$$
\mathcal{H}_{+} \subset \mathcal{H} \subset \mathcal{H}_{-}
$$

making it possible to construct a quasi-nucleous rigging of the dual Fock space $\Phi:=\oplus_{n \in \mathbb{Z}_{+}} \Phi_{(s)}^{\otimes n}$, where $\mathcal{H}_{+}$denotes the corresponding positive Hilbert space of testing functions. Thereby, the chain (14) generates the dual Fock space quasi-nucleous rigging

$$
\tilde{\mathcal{D}} \subset \Phi_{+} \subset \Phi \subset \Phi_{-} \subset \tilde{\mathcal{D}}^{\prime}
$$

with respect to the central Fock type Hilbert space $\Phi$, where $\tilde{\mathcal{D}} \simeq \mathcal{D}$, easily following from (1) and (14).

Construct now the following self-adjoint operator $\rho(x): \Phi \rightarrow \Phi$ as

$$
\rho(x):=a^{+}(x) a(x)
$$

called the density operator at a point $x \in \mathbb{R}^{m}$, satisfying the commutation properties:

$$
\begin{gathered}
{[\rho(x), \rho(y)]=0,} \\
{[\rho(x), a(y)]=-a(y) \delta(x-y),} \\
{\left[\rho(x), a^{+}(y)\right]=a^{+}(y) \delta(x-y)}
\end{gathered}
$$

for any $x, y \in \mathbb{R}^{m}$.

\subsection{The Unitary Family and Generating Functional Equations}

Suppose now that we have a continuous unitary representation of the unitary family $\mathcal{U}:=\{\exp [i \rho(f): f \in F\}$ in a suitable Hilbert space $\Phi$ with a cyclic vector $\mid \Omega) \in \Phi$. Then we can put, by definition,

$$
\mathcal{L}(f):=(\Omega|\mathrm{U}(f)| \Omega)
$$

for any $f \in F:=\mathcal{S}\left(\mathbb{R}^{m} ; \mathbb{R}\right)$, being the Schwartz space on $\mathbb{R}^{m}$, and observe that functional (18) is continuous on $F$ owing to the continuity of the representation. Therefore, this functional is the generalized Fourier transform of a cylindrical measure $\mu$ on $F^{\prime}$ :

$$
(\Omega|\mathrm{U}(f)| \Omega)=\int_{\mathcal{S}^{\prime}} \exp [i \eta(f)] d \mu(\eta) .
$$


From the spectral point of view, there is an isomorphism between the Hilbert spaces $\Phi$ and $L_{2}^{(\mu)}(F ; \mathbb{C})$, defined by $\left.\mid \Omega\right) \longrightarrow \Omega(\eta)=1$ and $\left.U(f) \mid \Omega\right) \longrightarrow \exp [i \eta(f)]$ and the extended by linearity upon the whole Hilbert space $\Phi$. Thus, having constructed the nonlinear functional (18) in an exact analytical form, one can retrieve the representation of the unitary family $\mathcal{U}$ in the corresponding Hilbert space $\Phi$ of the Fock type, as it follows from the expansion $\Phi=\oplus_{n \in \mathbb{Z}_{+}} \Phi_{n}$, where

$$
\left.\Phi_{n}=\underset{f_{n} \in L_{2,(s)}\left(\mathbb{R}^{m \times n} ; \mathbb{C}\right)}{\operatorname{span}}\left\{\prod_{j=1, n} a^{+}\left(x_{j}\right) \mid \Omega\right)\right\},
$$

for all $n \in \mathbb{Z}_{+}$.

The cyclic vector $\mid \Omega) \in \Phi$ can be, in particular, obtained as the ground state vector of some unbounded self-adjoint positive definite Hamilton operator $\mathrm{H}: \Phi \longrightarrow \Phi$, commuting with the self-adjoint non-negative particle number operator

$$
\mathrm{N}:=\int_{\mathbb{R}^{m}} \rho(x) d x,
$$

that is $[\mathrm{H}, \mathrm{N}]=0$. Moreover, the conditions

$$
\mathrm{H} \mid \Omega)=0
$$

and

$$
\inf _{\varphi \in D(\hat{\mathrm{H}})}(\varphi|\mathrm{H}| \varphi)=(\Omega|\mathrm{H}| \Omega)=0
$$

hold for the operator $\mathrm{H}: \Phi \longrightarrow \Phi$, where $D(\mathrm{H})$ denotes its domain of definition.

To find the functional (18), which is called the generating Bogolubov type functional for moment distribution functions

$$
F_{n}\left(x_{1}, x_{2}, \ldots, x_{n}\right):=\left(\Omega\left|: \rho\left(x_{1}\right) \rho\left(x_{2}\right) \ldots \rho\left(x_{n}\right):\right| \Omega\right),
$$

where $x_{j} \in \mathbb{R}^{m}, j=\overline{1, n}$, and the normal ordering operation : $:$ is defined $[1,3,14-17]$ as

$$
: \rho\left(x_{1}\right) \rho\left(x_{2}\right) \ldots \rho\left(x_{n}\right):=\prod_{j=1}^{n}\left(\rho\left(x_{j}\right)-\sum_{k=1}^{j-1} \delta\left(x_{j}-x_{k}\right)\right),
$$

it is convenient first to choose the Hamilton operator $\hat{H}: \Phi \longrightarrow \Phi$ in the following secondly quantized $[3,6,14,15]$ representation

$$
\mathrm{H}:=\frac{1}{2} \int_{\mathbb{R}^{m}}\left\langle\nabla_{x} a^{+}(x) \mid \nabla_{x} a(x)\right\rangle d x+\mathrm{V}(\rho),
$$

where the sign " $\nabla_{x}$ " means the usual gradient operation with respect to $x \in \mathbb{R}^{m}$ in the Euclidean space $\mathbb{R}^{m} \simeq\left(\mathbb{R}^{m} ;\langle\cdot \mid \cdot\rangle\right)$. If the energy spectrum density of the Hamiltonian operator (26) in the Hilbert space $\Phi$ is bounded from below, the expression (26) can be rewritten algebraically as

$$
\mathrm{H}=\frac{1}{2} \int_{\mathbb{R}^{m}}\left\langle\mathrm{~K}^{+}(x) \mid \rho^{-1}(x) \mathrm{K}(x)\right\rangle d x+\mathrm{V}(\rho),
$$

being equivalent in the Hilbert space $\Phi$, modulo the ground state energy eigenvalue, to the positive definite gauge type operator form

$$
\mathrm{H}=\frac{1}{2} \int_{\mathbb{R}^{m}}\left\langle\left(\mathrm{~K}^{+}(x)-\mathrm{A}(x ; \rho)\right) \mid \rho^{-1}(x)(\mathrm{K}(x)-\mathrm{A}(x ; \rho))\right\rangle d x,
$$


satisfying conditions (22) and (23), where $\mathrm{A}(x ; \rho): \Phi \rightarrow \Phi^{m}, x \in \mathbb{R}^{m}$, is some specially constructed linear self-adjoint operator, satisfying the condition

$$
\mathrm{K}(x) \mid \Omega)=\mathrm{A}(x ; \rho) \mid \Omega)
$$

for any $x \in \mathbb{R}^{m}$ and the ground state $\left.\mid \Omega\right) \in \Phi$, corresponding to a chosen potential operators $\mathrm{V}(\rho): \Phi \longrightarrow \Phi$. The "potential" operator $\mathrm{V}(\rho): \Phi \longrightarrow \Phi$ is, in general, a polynomial (or analytical) functional of the density operator $\rho(x): \Phi \longrightarrow \Phi$ for any $x \in \mathbb{R}^{m}$, and the operator $\mathrm{K}(x): \Phi \longrightarrow \Phi^{m}$ is given as

$$
\mathrm{K}(x):=\nabla_{x} \rho(x) / 2+i J(x),
$$

where the self-adjoint "current" operator $J(x): \Phi \longrightarrow \Phi^{m}$ can be naturally defined (but non-uniquely) from the continuity equality

$$
\partial \rho / \partial t=-i[\mathrm{H}, \rho(x)]=-<\nabla \mid J(x)>,
$$

holding for all $x \in \mathbb{R}^{m}$. Such an operator $J(x): \Phi \longrightarrow \Phi^{m}, x \in \mathbb{R}^{m}$, can exist owing to the commutation condition $[\hat{\mathrm{H}}, \hat{\mathrm{N}}]=0$, giving rise to the continuity relationship (31), if additionally to take into account that supports supp $\rho$ of the density operator $\rho(x): \Phi \longrightarrow \Phi, x \in \mathbb{R}^{m}$, can be chosen arbitrarily owing to the independence of (31) on the potential operator $V(\rho): \Phi \longrightarrow \Phi$, but its strict dependence on the corresponding representation (19). In particular, based on the Fock space $\Phi$, defined by (1) and generated by the creation-annihilation operators (7) and (8), the current operator $J(x): \Phi \longrightarrow \Phi^{m}$, $x \in \mathbb{R}^{m}$, can be easily constructed as follows:

$$
J(x)=\frac{1}{2 i}\left[a^{+}(x) \nabla_{x} a(x)-\nabla_{x} a^{+}(x) a(x)\right],
$$

satisfying jointly with the density operator $\rho(x): \Phi \longrightarrow \Phi, x \in \mathbb{R}^{m}$, defined by (16), the following quantum current symmetry algebra $[6,7,14-18]$ relationships:

$$
\begin{aligned}
& {\left[J\left(g_{1}\right), J\left(g_{2}\right)\right]=i J\left(\left[g_{1}, g_{2}\right]\right),\left[\rho\left(f_{1}\right), \rho\left(f_{2}\right)\right]=0,} \\
& {\left[J\left(g_{1}\right), \rho\left(f_{1}\right)\right]=i \rho\left(\left\langle g_{1} \mid \nabla f_{1}\right\rangle\right),}
\end{aligned}
$$

holding for all $f_{1}, f_{1} \in F$ and $g_{1}, g_{2} \in F^{m}$, where we put, by definition,

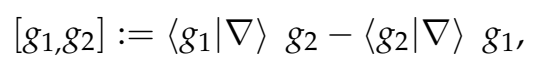

being the usual commutator of vector fields $\left\langle g_{1} \mid \nabla\right\rangle$ and $\left\langle g_{2} \mid \nabla\right\rangle$ on the configuration space $\mathbb{R}^{m}$. It is easy to observe that the current algebra (33) is the Lie algebra $\mathcal{G}$, corresponding to the Banach Lie group $G=\operatorname{Diff}\left(\mathbb{R}^{m}\right) \ltimes F$, the semidirect product of the Banach Lie group of diffeomorphisms Diff $\left(\mathbb{R}^{m}\right)$ of the $m$-dimensional space $\mathbb{R}^{m}$ and the abelian group $F$ subject to the multiplicative operation Banach group of smooth functions $F$.

Remark 1. The self-adjointness of the operator $\mathrm{A}(g ; \rho): \Phi \longrightarrow \Phi, g \in F$, can be stated following schemes from works $[6,14,18]$ under the additional existence of such a linear anti-unitary mapping $\mathrm{T}: \Phi \longrightarrow \Phi$ that the following invariance conditions hold:

$$
\left.\left.\mathrm{T} \rho(x) \mathrm{T}^{-1}=\rho(x), \quad \mathrm{T} J(x) \mathrm{T}^{-1}=-J(x), \quad \mathrm{T} \mid \Omega\right)=\mid \Omega\right)
$$

for any $x \in \mathbb{R}^{m}$. Thereby, owing to conditions (35), the following equalities

$$
\mathrm{K}(x) \mid \Omega)=\mathrm{A}(x ; \rho) \mid \Omega)
$$


hold for any $x \in \mathbb{R}^{m}$, giving rise to the self-adjointness of the operator $\mathrm{A}(g ; \rho): \Phi \longrightarrow \Phi, g \in \mathbb{F}$.

It is easy to observe that the time-reversal condition (35) imposes the real value relationship for the ground state $\Omega_{N}=\bar{\Omega}_{N} \in \Phi_{N} \simeq L_{2}^{(s)}\left(\mathbb{R}^{m \times N} ; \mathbb{C}\right)$ of the canonically represented $N$-particle Hamiltonian $\mathrm{H}_{N}: \Phi_{N} \rightarrow \Phi_{N}$ for arbitrary $N \in \mathbb{Z}_{+}$. Moreover, taking into account the relationship (36), one can easily observe that on the invariant subspace $\Phi_{N} \subset \Phi$ the operator $K(x): \Phi \longrightarrow \Phi$ is representable as

$$
K_{N}(x)=\sum_{j=\overline{1, N}} \delta\left(x-x_{j}\right) \frac{\partial}{\partial x_{j}}
$$

entailing the following expression for the related operator $A_{N}(x ; \rho): \Phi_{N} \rightarrow \Phi_{N}$ on the subspace $\Phi_{N} \subset \Phi:$

$$
A_{N}(x ; \rho)=\sum_{j=\overline{1, N}} \delta\left(x-x_{j}\right) \nabla_{x_{j}} \ln \left|\Omega_{N}\left(x_{1}, x_{2}, \ldots, x_{N}\right)\right| .
$$

The latter makes it possible to derive its secondly quantized [2,3] expression as

$$
\mathrm{A}(x ; \rho)=\int_{\mathbb{R}^{m \times N}} d x_{2} d x_{3} \ldots d x_{N}: \rho(x) \rho\left(x_{2}\right) \rho\left(x_{3}\right) \ldots \rho\left(x_{N}\right): \nabla_{x} \ln \left|\Omega_{N}\left(x, x_{2}, \ldots, x_{N}\right)\right|,
$$

which holds for any $x \in \mathbb{R}^{m}$ and arbitrary $N \in \mathbb{Z}_{+}$. Being interested in the infinite particle case when $N \rightarrow \infty$, the expression (39) can be naturally decomposed $[19,20]$ as

$$
\mathrm{A}(x ; \rho)=\sum_{n \in \mathbb{Z}_{+}} \frac{1}{n !} \int_{\mathbb{R}^{m \times n}} d y_{1} d y_{2} \ldots d y_{n}: \rho(x) \rho\left(y_{1}\right) \rho(y) \rho\left(y_{3}\right) \ldots \rho\left(y_{n}\right): \nabla_{x} W_{n+1}\left(x ; y_{1}, y_{2}, \ldots, y_{n}\right),
$$

where the corresponding real-valued coefficients $W_{n} \in H_{2}^{(1)}\left(\mathbb{R}^{m \times n} ; \mathbb{R}\right)$ should be such functions that the series (40) were convergent in a suitably chosen representation Fock space $\Phi$, for which the resulting ground state $\left.\lim _{N \rightarrow \infty} \Omega_{N} \simeq \mid \Omega\right) \in \Phi$ is necessarily cyclic and normalized.

Based now on the construction above one easily deduces from expression (30) that the generating Bogolubov type functional (18) obeys for all $x \in \mathbb{R}^{m}$ the following functional-differential equation:

$$
\left[\nabla_{x}-i \nabla_{x} f\right] \frac{1}{i} \frac{\delta \mathcal{L}(f)}{\delta f(x)}=\mathrm{A}\left(x ; \frac{1}{i} \frac{\delta}{\delta f}\right) \mathcal{L}(f),
$$

whose solutions should satisfy the Fourier transform representation (19). In particular, a wide class of special so-called Poissonian white noise type solutions to the functional-differential equation (41) was obtained in $[6,9,10,14,21,22]$ by means of functional-operator methods in the following generalized form:

$$
\mathcal{L}(f)=\exp \left\{\int_{\mathbb{R}^{m}} \mathrm{~W}\left(\frac{1}{i} \frac{\delta}{\delta f}\right) d x\right\} \exp \left(\bar{\rho} \int_{\mathbb{R}^{m}}\{\exp [i f(x)]-1\} d x\right),
$$

where $\bar{\rho}=(\Omega|\rho| \Omega) \in \mathbb{R}_{+}$is a suitable Poisson process parameter and the operator $\mathrm{A}(x ; \rho): \Phi \rightarrow$ $\Phi^{m}, x \in \mathbb{R}^{m}$, is suitably represented as

$$
\mathrm{A}(x ; \rho)=\rho(x) \nabla \frac{\delta}{\delta \rho(x)} \mathrm{W}(\rho),
$$

resulting from the expression (40) for some scalar operator $\mathrm{W}(\rho): \Phi \rightarrow \Phi$.

Remark 2. It is worth to remark here that solutions to Equation (41) realize the suitable physically motivated representations of the abelian Banach subgroup F of the Banach group $G=\operatorname{Diff}\left(\mathbb{R}^{m}\right) \ltimes F$, mentioned above. In the general case of this Banach group $G$ one can also construct $[6,14,16,23]$ a generalized Bogolubov 
type functional equation, whose solutions give rise to suitable physically motivated representations of the corresponding current Lie algebra $\mathcal{G}$.

Recalling now the Hamiltonian operator representation (28), one can readily deduce that the following weak representation Hilbert space $\Phi$ weak relationship

$$
\left(\left\langle\mathrm{A} \mid \rho^{-1} \mathrm{~A}\right\rangle-\left\langle\mathrm{K}^{*} \mid \rho^{-1} \mathrm{~A}\right\rangle-\left\langle\mathrm{A} \mid \rho^{-1} \mathrm{~K}\right\rangle\right) / 2-\mathrm{V}(\rho)=\epsilon_{0},
$$

where $\epsilon_{0} \in \mathbb{R}$ is the corresponding ground state energy density value. Thus, the main analytical problem is now reduced to constructing the expansion (40) corresponding to a suitable cyclic representation Hilbert space $\Phi$ of the quantum current algebra (33), compatible with the Hamiltonian operator structure (27).

\section{The Canonical Representation of the Nonrelativistic Local Current Algebra and the Factorized Structure of Quantum Integrable Many-Particle Hamiltonian Systems}

\subsection{The Density Functional Representation of the Current Algebra}

We are now interested in constructing the density functional representation of the current algebra (33) in the Hilbert space $\Phi \simeq \mathcal{H}_{\rho}$ with the cyclic vector $\left.\mid \Omega\right)=1 \in \Phi$. To do this, let us consider first the "creation" $a^{+}(x)$ and "annihilation" operators $a(x), x \in \mathbb{R}^{m}$, defined via (31) in the canonical Fock space $\Phi$, which can be formally represented as

$$
a^{+}(x)=\sqrt{\rho(x)} \exp [-i \vartheta(x)], a(x)=\exp [i \vartheta(x)] \sqrt{\rho(x)},
$$

where $\rho(x): \Phi \rightarrow \Phi$ is our density operator and $\vartheta(x): \Phi \rightarrow \Phi, x \in \mathbb{R}^{m}$, is some self-adjoint operator. What is important, the operators $\rho(x)$ and $\vartheta(x): \Phi \rightarrow \Phi$ realize the canonical commutation relationships

$$
\begin{aligned}
& {[\rho(x), \rho(y)]=0=[\vartheta(x), \vartheta(y)]} \\
& {[\rho(y), \vartheta(x)]=i \delta(x-y)}
\end{aligned}
$$

for any $x, y \in \mathbb{R}^{m}$. Concerning the current operator $J(x): \Phi \rightarrow \Phi, x \in \mathbb{R}^{m}$, one can easily obtain its equivalent expression

$$
J(x)=\rho(x) \nabla \vartheta(x) .
$$

Based on the canonical relationships (46) one can easily obtain, following [18], that

$$
\vartheta(x)=\frac{1}{i} \frac{\delta}{\delta \rho(x)}+i \sigma[\rho(x)],
$$

where $\sigma[\rho(x)]: \Phi \rightarrow \Phi$ is some function of the density operator $\rho(x): \Phi \rightarrow \Phi, x \in \mathbb{R}^{m}$. Then, respectively, the current operator (48) is representable in $\Phi$ as

$$
J(x)=-i \rho(x) \nabla \frac{\delta}{\delta \rho(x)}+\rho(x) \nabla \sigma[\rho(x)] .
$$

The functional-operator expression (50) proves to make sense $[6,18,24]$ as operators in the Hilbert space $\mathcal{H}_{\rho} \simeq \Phi$ of functional valued complex-functions on the manifold $\mathcal{M}$, coordinated by the density parameter $\rho: \Phi \rightarrow \Phi$ and endowed with the scalar product $(a \mid b)_{\mathcal{H}_{\rho}}:=\int_{\mathcal{M}} \overline{a(\rho)} b(\rho) d \mu(\rho)$ subject to some measure $\mu$ on $\mathcal{M}$. To calculate this measure $\mu$ on $\mathcal{M}$, we will present an explicit isomorphism 
between this Hilbert space $\mathcal{H}_{\rho}$ and the corresponding Fock space $\Phi$ of spinless bosonic particles in $\mathbb{R}^{m}$. First, we determine the support supp $\mu \subset \mathcal{M}$ of the measure $\mu$, having assumed that the manifold

$$
\mathcal{M}=\cup_{n \in \mathbb{Z}_{+}} \mathcal{M}_{n}
$$

where $\mathcal{M}_{n}:=\left\{a(\rho): \rho(x):=\sum_{j=1}^{n} \delta\left(x-c_{j}\right): a \in C^{\infty}\left(F^{\prime} ;\right.\right.$ End $\left.\left.\mathcal{H}_{\rho}\right)\right\}$, where $c_{j} \in \mathbb{R}^{m}, j=\overline{1, n}, n \in \mathbb{N}$, are arbitrary vector parameters. The restriction $d \mu_{n}$ of the measure $\mu$ on the submanifold $\mathcal{M}_{n}$ can be presented $[2,6,14,15,21]$ as

$$
d \mu_{n}=\gamma_{n}\left(c_{1}, c_{2}, \ldots, c_{n}\right) \prod_{j=1, n} d c_{j}
$$

where functions $\gamma_{n}: \mathbb{R}^{m \times n} \rightarrow \mathbb{R}_{+}, n \in \mathbb{N}$, should be determined from the condition (50). In accordance with the manifold structure (51) we can decompose the Hilbert space $\mathcal{H}_{\rho}$ as

$$
\mathcal{H}_{\rho}=\oplus_{n \in \mathbb{N}} \mathcal{H}_{n}
$$

where the space $\mathcal{H}_{n}$ depends on the mapping $\sigma: \mathcal{M} \rightarrow \operatorname{End}\left(\mathcal{H}_{\rho}\right)$ and consists of functionals that are bounded on $\mathcal{M}_{n}$, in particular, for any $a(\rho) \in \mathcal{M}$ the restrictions $\left.a(\rho)\right|_{\mathcal{H}_{n}}, n \in \mathbb{N}$, consist of functions of vectors $\left(c_{1}, c_{2}, \ldots, c_{n}\right) \in \mathbb{R}^{m \times n}, n \in \mathbb{N}$, respectively. The scalar product in $\mathcal{H}_{n}, n \in \mathbb{N}$, is suitably defined by means of the expressions (52). Now we can construct the isomorphism between the Hilbert spaces $\mathcal{H}_{n}, n \in \mathbb{N}$, and the corresponding components $\Phi_{n}, n \in \mathbb{N}$, of the corresponding Fock space $\Phi$, representing spinless bosonic particles in $\mathbb{R}^{m}$. In the Hilbert space $\mathcal{H}_{n}:=\mathcal{H}_{n}^{(\sigma)}, n \in \mathbb{N}$, one can easily calculate the eigenfunctions $\varphi_{p_{1}, p_{2}, \ldots, p_{n}}^{(\sigma)}(\rho) \in \mathcal{H}_{n}^{(\sigma)}$ of the free Hamiltonian

$$
\mathrm{H}_{0}^{(\sigma)}:=\frac{1}{2} \int_{\mathbb{R}^{m}} d x<K^{+}(x) \mid \rho^{-1}(x) K(x)>
$$

with structural

$$
K(x):=\frac{1}{2} \nabla \rho(x)+i J^{(\sigma)}(x), \quad K^{+}(x):=\frac{1}{2} \nabla \rho(x)-i J^{(\sigma)}(x)
$$

and the momentum operators

$$
\begin{aligned}
\mathrm{P}^{(\sigma)}:= & \int_{\mathbb{R}^{m}} d x J^{(\sigma)}(x), \\
\mathrm{H}_{0}^{(\sigma)} \varphi_{p_{1}, p_{2}, \ldots, p_{n}}^{(\sigma)}(\rho) & =\left(\sum_{j=\overline{1, n}} E_{j}\right) \varphi_{p_{1}, p_{2}, \ldots, p_{n}}^{(\sigma)}(\rho), \\
\mathrm{P}^{(\sigma)} \varphi_{p_{1}, p_{2}, \ldots, p_{n}}^{(\sigma)}(\rho) & =\left(\sum_{j=\overline{1, n}} p_{j}\right) \varphi_{p_{1}, p_{2,}, \ldots, p_{n}}^{(\sigma)}(\rho),
\end{aligned}
$$

where $p_{j} \in \mathbb{R}^{m}, j=\overline{1, n}$, are momentums of bose-particles in $\mathbb{R}^{m}$, the operator $\mathrm{H}_{0}^{(\sigma)}: \mathcal{H}_{\rho} \rightarrow \mathcal{H}_{\rho}$ is given by the expressions (54), (30) and (50) and the operator $\mathrm{P}^{(\sigma)}: \mathcal{H}_{\rho} \rightarrow \mathcal{H}_{\rho}$ is given by the expressions (55) and (50), respectively, within which the current operator $J^{(\sigma)}(x): \mathcal{H}_{\rho} \rightarrow \mathcal{H}_{\rho}$ is realized under the condition $\nabla \sigma[\rho(x)]:=\sigma \rho(x)^{-1} \nabla \rho(x)$ as

$$
J^{(\sigma)}(x)=-i \rho(x) \nabla \frac{\delta}{\delta \rho(x)}+i \sigma \nabla \rho(x)
$$

where $\sigma \in \mathbb{R}$ is a fixed real-valued parameter. In this case the eigenfunctions $\varphi_{p_{1}, p_{2}, \ldots, p_{n}}^{(\sigma)}(\rho) \in \mathcal{H}_{n}^{(\sigma)}, n \in$ $\mathbb{N}$, can be expressed $[18,25]$ as

$$
\varphi_{p_{1}, p_{2}, \cdots, p_{n}}^{(\sigma)}(\rho)=\frac{1}{n !} \bar{\varphi}_{0}^{(\sigma)}(\rho)\left(\prod_{j=1, n} B_{p_{j}}(\rho) \cdot 1\right),
$$


where

$$
\begin{aligned}
& \bar{\varphi}_{0}^{(\sigma)}(\rho):=\exp \left[(\sigma-1 / 2) \int_{\mathbb{R}^{m}} d x \rho(x) \ln \rho(x)\right] \\
& B_{p_{j}}(\rho):=\int_{\mathbb{R}^{m}} d x \exp (i<p \mid x>) \rho(x) \exp \left(-\frac{\delta}{\delta \rho(x)}\right)
\end{aligned}
$$

The corresponding $n$-particle Fock subspaces $\Phi_{n}^{(\sigma)}, n \in \mathbb{N}$, can be naturally represented by means of the vectors

$$
\left.\left.\mid \varphi_{n}^{(\sigma)}\right):=\frac{1}{\sqrt{n !}} \int_{\mathbb{R}^{m \times n}} \prod_{j=1, n} d p_{j} f_{n}^{(\sigma)}\left(p_{1}, p_{2}, \ldots, p_{n}\right) a^{+}\left(p_{1}\right) a^{+}\left(p_{2}\right) \ldots a^{+}\left(p_{n}\right) \mid \Omega_{0}\right)
$$

with functions $f_{n}^{(\sigma)} \in L_{2}^{(s)}\left(\mathbb{R}^{m \times n} ; \mathbb{C}\right), n \in \mathbb{N}$, where

$$
a^{+}(p):=\frac{1}{(2 \pi)^{m / 2}} \int_{\mathbb{R}^{m}} d x \exp (i<x \mid p>) a^{+}(x)
$$

denotes the momentum creation operator for any $p \in \mathbb{R}^{m}$.

Moreover, any functional $\varphi_{n}^{(\sigma)}(\rho) \in \mathcal{H}_{n}^{(\sigma)}, n \in \mathbb{N}$, can be uniquely represented as

$$
\varphi_{n}^{(\sigma)}(\rho):=\int_{\mathbb{R}^{m \times n}} \prod_{j=1, n} d p_{j} f_{n}^{(\sigma)}\left(p_{1}, p_{2}, \ldots, p_{n}\right) \varphi_{p_{1}, p_{2}, \ldots, p_{n}}^{(\sigma)}(\rho)
$$

for $f_{n}^{(\sigma)} \in L_{2}^{(s)}\left(\mathbb{R}^{m \times n} ; \mathbb{C}\right)$, since the following condition

$$
\left.\left.\left(B_{p_{n+1}}(\rho) \prod_{j=1, n} B_{p_{j}}(\rho) \cdot 1\right)\right|_{\rho=a^{+}(x) a(x)} \mid \varphi_{n}^{(\sigma)}\right)=0
$$

holds identically for all $p_{j} \in \mathbb{R}^{m}, j=\overline{1, n+1}$, and arbitrary state $\left.\mid \varphi_{n}^{(\sigma)}\right) \in \Phi, n \in \mathbb{N}$.

Remark 3. The condition (64) jointly with the constraint $\int_{\mathbb{R}^{m}} \rho(x) d x=n$ in $\Phi_{n}^{(\sigma)}, n \in \mathbb{N}$, should be, in general, naturally satisfied for any current algebra representation space $\Phi$ if and only if $\rho(x)=\sum_{j=1, n} \delta(x-$ $\left.c_{j}\right) \in \mathcal{M}_{n}$ for arbitrary $n \in \mathbb{N}$.

As a result of the construction above we can state that the Hilbert spaces $\mathcal{H}_{n}^{(\sigma)}, n \in \mathbb{N}$, and Fock subspaces $\Phi_{n}^{(\sigma)}, n \in \mathbb{N}$, are, respectively, isomorphic. As a consequence we derive that the Hilbert space $\mathcal{H}_{\rho}$ and the Fock space $\Phi$ are isomorphic too.

Consider now, following $[6,18]$, the action of the current operator $(58)$ on the basic vectors $\varphi_{n}^{(\sigma)}(\rho) \in \mathcal{H}_{n}^{(\sigma)}, n \in \mathbb{N}:$

$$
J^{(\sigma)}(x) \varphi_{n}^{(\sigma)}(\rho)=\bar{\varphi}_{0}^{(\sigma)}(\rho)\left[-i \rho(x) \nabla \frac{\delta}{\delta \rho(x)}+i \sigma \nabla \rho(x)\right] \varphi_{n}^{(\sigma)}(\rho)
$$

from which one ensues easily at $\sigma=1 / 2$ its $n$-particle representation on the functional manifold $\mathcal{M}_{n}$ :

$$
\begin{gathered}
\left.J^{(1 / 2)}(x) \varphi_{n}^{(1 / 2)}(\rho)\right|_{\rho(y)=\sum_{j=\overline{1, n}} \delta\left(y-c_{j}\right)}= \\
=\sum_{j=\overline{1, n}} \frac{1}{2}\left[-i \delta\left(x-c_{j}\right) \nabla_{c_{j}}+i \nabla_{c_{j}} \circ \delta\left(x-c_{j}\right)\right] \tilde{f}_{n}^{(1 / 2)}\left(c_{1}, c_{2}, \ldots, c_{n}\right),
\end{gathered}
$$


where we took into account that $\bar{\varphi}_{0}^{(1 / 2)}(\rho)=1$ for all densities $\rho: \Phi \rightarrow \Phi$ and have put, by definition, the Fourier transform

$$
\tilde{f}_{n}^{(1 / 2)}\left(c_{1}, c_{2}, \ldots, c_{n}\right):=\int_{\mathbb{R}^{m \times n}} \prod_{j=1, n} d p_{j} f_{n}^{(1 / 2)}\left(p_{1}, p_{2}, \ldots, p_{n}\right) \exp \left(i \sum_{j=\overline{1, n}}<p_{j} \mid c_{j}>\right)
$$

for any fixed particle position vectors $c_{j} \in \mathbb{R}^{n}, j=\overline{1, n}$, and for arbitrary $n \in \mathbb{N}$. The expression (66), in particular, means that the current operator $J^{(1 / 2)}(x): \mathcal{H}_{\rho} \rightarrow \mathcal{H}_{\rho}$ is symmetric with respect to the measure $d \mu_{n}^{(1 / 2)}:=\beta_{n} \prod_{j=\overline{1, n}} d c_{j}$ on each functional submanifold $\mathcal{M}^{n}$ for all $n \in \mathbb{N}$, where the constants $\beta_{n} \in \mathbb{R}_{+}, n \in \mathbb{N}$, can be determined from the normalization condition $\left\|\varphi_{n}^{(1 / 2)}(\rho)\right\|_{\mathcal{H}_{n}^{(1 / 2)}}=\left(\varphi_{n}^{(1 / 2)} \mid \varphi_{n}^{(1 / 2)}\right)_{\Phi_{n}^{(1 / 2)}}^{1 / 2}, n \in \mathbb{N}$. The latter gives rise $[2,14,15,21]$ to the following symbolic measure expression

$$
d \mu_{n}^{(1 / 2)}:=\prod_{x \in \mathbb{R}^{m}} \delta\left(\rho(x)-\sum_{j=1, n} \delta\left(x-c_{j}\right)\right) \prod_{j=1, n} \frac{d c_{j}}{(2 \pi)^{m}}
$$

for all $c_{j} \in \mathbb{R}^{n}, j=\overline{1, n}$, and arbitrary $n \in \mathbb{N}$.

Remark 4. As was aptly observed in [18], the choice $\sigma=1 / 2$ makes it possible to realize the current algebra representation in the space $\mathcal{M}$ of analytic functions, what will be a priori assumed for further, that is the corresponding measure can be symbolically expressed as

$$
d \mu_{n}:=\prod_{x \in \mathbb{R}^{m}} \delta\left(\rho(x)-\sum_{j=\overline{1, n}} \delta\left(x-c_{j}\right)\right) \prod_{j=1, n} \frac{d c_{j}}{(2 \pi)^{n}}
$$

on the subspace $\mathcal{M}_{n}$ for any $n \in \mathbb{N}$.

3.2. The Generalized Quantum Oscillatory Model: The Density Functional Current Algebra Representation and the Hamiltonian Reconstruction

As a classical application of the construction above one can consider a current algebra representation of the quantum Hamiltonian operator

$$
\mathrm{H}^{(\omega)}=\frac{1}{2} \int_{\mathbb{R}^{m}}<K(x)^{+}\left|\rho(x)^{-1} K(x)>d x+\frac{1}{2} \int_{\mathbb{R}^{m}}<\omega x\right| \omega x>\rho(x) d x
$$

in the corresponding Fock space $\Phi$ of the generalized quantum $N$-particle oscillatory Hamiltonian

$$
H_{N}^{(\omega)}=\frac{1}{2} \sum_{j=1, N}\left(<\nabla_{x_{j}}\left|\nabla_{x_{j}}>+<\omega x_{j}\right| \omega x_{j}>\right)
$$

for $N \in \mathbb{Z}_{+}$bose-particles in the $m$-dimensional space $\mathbb{R}^{m}$ under the external oscillatory potential, parametrized by the positive definite frequency matrix $\omega \in E n d \mathbb{R}^{m}$.

Having shifted the representation Hilbert space $\mathcal{H}_{\rho}$ by the functional $\bar{\varphi}_{0}^{(1 / 2)}(\rho):=$ $\exp \left[-\frac{1}{2} \int_{\mathbb{R}^{m}}\langle x \mid \omega x\rangle \rho(x) d x\right] \in \mathcal{H}_{\rho}$, the corresponding current operator (58) becomes

$$
J^{(\omega)}(x)=-i \rho(x) \nabla \frac{\delta}{\delta \rho(x)}+\frac{i}{2} \nabla \rho(x)-i \omega x \rho(x),
$$


entailing simultaneously the related $K$-operator changing

$$
K(x)=\rho(x) \nabla \frac{\delta}{\delta \rho(x)} \rightarrow K^{(\omega)}(x)=\rho(x) \nabla \frac{\delta}{\delta \rho(x)}+\omega x \rho(x)
$$

for any $x \in \mathbb{R}^{m}$. The latter gives rise, respectively, to the following equivalent current algebra functional representation of the oscillatory Hamiltonian (70):

$$
\mathrm{H}^{(\omega)}=\frac{1}{2} \int_{\mathbb{R}^{m}}<K^{(\omega)}(x)^{+} \mid \rho(x)^{-1} K^{(\omega)}(x)>d x+\frac{1}{2} \operatorname{tr} \omega \int_{\mathbb{R}^{m}} \rho(x) d x
$$

for any pisitive define matrix $\omega \in E n d \mathbb{R}^{m}$. The shifted current operator (72) makes it possible to construct the suitably deformed free particle measure

$$
d \mu_{1}^{(\omega)}(\rho):=\exp \left(-\int_{\mathbb{R}^{m}} d x \rho(x)\langle x \mid \omega x\rangle\right) d \mu_{1}^{(1 / 2)}(\rho)
$$

on the one-particle functional manifold $\mathcal{M}_{1}$, for which the following expression

$$
\left(\Omega\left|\mathrm{H}^{(\omega)}\right| \mathrm{U}(f) \mid \Omega\right)=\int_{\mathcal{M}} \exp [i \rho(f)] d \mu_{1}^{(\omega)}(\rho)
$$

holds for any test function $f \in F$. The latter, jointly with the related ground state condition $\mid \Omega)=1 \in \mathcal{H}_{\rho}$, makes it possible to calculate easily the scalar product elements

$$
\left(\mathrm{U}\left(f_{1}\right) \Omega\left|\mathrm{H}^{(\omega)}\right| \mathrm{U}\left(f_{2}\right) \mid \Omega\right)=\int_{\mathbb{R}^{m}} \exp \left[i f_{1}(c)+i f_{2}(c)\right] \exp (-\langle c \mid \omega c\rangle) \frac{d c}{(2 \pi)^{m}}
$$

for any test functions $f_{1}, f_{2} \in F$. The expression (77) makes it possible to calculate successfully the matrix elements $\left(\rho\left(f_{p_{1}}\right) \Omega\left|\mathrm{H}^{(\omega)}\right| \rho\left(f_{p_{2}}\right) \mid \Omega\right)$ of the Hamiltonian $\mathrm{H}^{(\omega)}: \mathcal{H}_{\rho} \rightarrow \mathcal{H}_{\rho}$ on the corresponding eigenvectors $\left.\rho\left(f_{p}\right) \mid \Omega\right) \in \mathcal{H}_{\rho}$ for arbitrary $p=p_{1}, p_{2} \in \mathbb{N}$ and, therefore, to find its spectrum.

Consider now the operator (30) taking into account the analytical current representation (65) at $\sigma=1 / 2$ :

$$
\begin{gathered}
K(x) \varphi_{n}^{(1 / 2)}(\rho)=\left[\rho(x) \nabla_{\frac{\delta}{\delta \rho(x)}}-1 / 2 \nabla \rho(x)\right] \varphi_{n}^{(1 / 2)}(\rho)+ \\
+1 / 2 \nabla \rho(x) \varphi_{n}^{(1 / 2)}(\rho)=\rho(x) \nabla \frac{\delta}{\delta \rho(x)} \varphi_{n}^{(1 / 2)}(\rho)
\end{gathered}
$$

for any $n \in \mathbb{N}$. Having substituted instead of $\varphi_{n}^{(1 / 2)}(\rho) \in \mathcal{H}_{\rho}^{(n)}, n \in \mathbb{N}$, the ground state eigenfunction $\Omega(\rho)=1 \in \mathcal{H}_{\rho}$, we can easily retrieve the before derived expression (39). Moreover, based on the representation (73) and the definition (29), one can calculate that

$$
\begin{aligned}
K^{(\omega)}(x) \bar{\varphi}_{0}^{(1 / 2)}(\rho) & =\left[\rho(x) \nabla \frac{\delta}{\delta \rho(x)}+\omega x \rho(x)\right] \bar{\varphi}_{0}^{(1 / 2)}(\rho)=0= \\
& =A^{(\omega)}(x ; \rho) \bar{\varphi}^{(1 / 2)}(\rho),
\end{aligned}
$$

where $\bar{\varphi}_{0}^{(1 / 2)}(\rho)=\exp \left[-\frac{1}{2} \int_{\mathbb{R}^{m}}\langle x \mid \omega x\rangle \rho(x) d x\right] \in \mathcal{H}_{\rho}^{(1 / 2)} \simeq \mathcal{H}_{\rho}$. The latter means, in particular, that the corresponding multiplication operator $A^{(\omega)}(x ; \rho)=0$, or, respectively,

$$
K(x) \bar{\varphi}_{0}^{(1 / 2)}(\rho):=A(x ; \rho) \bar{\varphi}_{0}^{(1 / 2)}(\rho)=-\omega x \rho(x) \bar{\varphi}_{0}^{(1 / 2)}(\rho),
$$


where $\left.\bar{\varphi}_{0}^{(1 / 2)}(\rho):=\mid \Omega(\rho)\right) \in \mathcal{H}_{\rho}$ is the corresponding ground state vector in $\mathcal{H}_{\rho}$ for the oscillatory Hamiltonian operator (71). Making use of the operator (75), based on expression (42) one can present a special solution to the functional Equation (41) in the form

$$
\mathcal{L}(f)=\exp \left(-\int_{\mathbb{R}^{m}} d x\langle\omega x \mid x\rangle \frac{1}{2 i} \frac{\delta}{\delta f(x)}\right) \exp \left(\bar{\rho} \int_{\mathbb{R}^{m}}\{\exp [i f(x)]-1\} d x\right),
$$

confirming similar statements from $[6,16,20]$.

3.3. The Calogero-Sutherland Quantum Model: The Current Algebra Representation, the Hamiltonian Reconstruction and Integrability

The periodic Calogero-Sutherland quantum bosonic model on the finite interval $[0, l] \simeq \mathbb{R} /[0, l] \mathbb{Z}$ is governed by the $N$-particle Hamiltonian

$$
H_{N}:=-\sum_{j=1, N} \frac{\partial^{2}}{\partial x_{j}^{2}}+\sum_{j \neq k=1, N} \frac{\pi^{2} \beta(\beta-1)}{l^{2} \sin ^{2}\left[\frac{\pi}{l}\left(x_{j}-x_{k}\right)\right]}
$$

in the symmetric Hilbert space $L_{2}^{(s)}\left([0, l]^{N} ; \mathbb{C}\right)$, where $N \in \mathbb{Z}_{+}$and $\beta \in \mathbb{R}$ is an interaction parameter. As it was stated in a very interesting and highly speculative work [26], there exists linear differential operators

$$
\mathcal{D}_{j}:=\frac{\partial}{\partial x_{j}}-\frac{\pi \beta}{l} \sum_{k=1, N, k \neq j} \operatorname{ctg}\left[\frac{\pi}{l}\left(x_{j}-x_{k}\right)\right]
$$

for $j=\overline{1, N}$, such that the Hamiltonian (83) is factorized as the bounded from below symmetric operator

$$
H_{N}=\sum_{j=\overline{1, N}} \mathcal{D}_{j}^{+} \mathcal{D}_{j}+E_{n}
$$

where

$$
E_{N}=\frac{1}{3}\left(\frac{\pi \beta}{l}\right)^{2} N\left(N^{2}-1\right)
$$

is the ground state energy of of the Hamiltonian operator (83), that is there exists such a vector $\left.\mid \Omega_{N}\right) \in L_{2}^{(s)}\left([0, l]^{N} ; \mathbb{C}\right)$, satisfying for any $N \in \mathbb{Z}_{+}$the eigenfunction condition

$$
\left.\left.H_{N} \mid \Omega_{N}\right)=E_{N} \mid \Omega_{N}\right)
$$

and equals

$$
\left.\mid \Omega_{N}\right)=\prod_{j<k=1, N}\left(\sin \left[\frac{\pi}{l}\left(x_{j}-x_{k}\right)\right]\right)^{\beta} .
$$

Being interested additionally in proving the quantum integrability of the Calogero-Sutherland model (83), we will proceed to its second quantized representation [2,3] and studying it by means of the current algebra representation approach, described above in Section 2.1 and devised before in $[6,15-17,20,27]$.

The secondly quantized form of the Calogero-Sutherland Hamiltonian operator (83) looks as

$$
\mathrm{H}=\int_{0}^{l} d x \psi_{x}^{+}(x) \psi_{x}(x)+\left(\frac{\pi}{l}\right)^{2} \beta(\beta-1) \int_{0}^{l} d x \int_{0}^{l} d y \frac{\psi^{+}(x) \psi^{+}(y) \psi(y) \psi(x)}{\sin ^{2}\left[\frac{\pi}{l}(x-y)\right]}
$$

acting in the corresponding Fock space $\Phi:=\oplus_{n \in \mathbb{Z}_{+}} \Phi_{n}, \Phi_{n} \simeq L_{2}^{(s)}\left([0, l]^{n} ; \mathbb{C}\right), n \in \mathbb{Z}_{+}$. To proceed to the current algebra representation of the Hamiltonian operator (89), it would useful to recall the 
factorized representation (85) and construct preliminarily the following singular Dunkl type [28] symmetrized differential operator

$$
\begin{gathered}
D_{N}(x):=\sum_{j=\overline{1, N}} \delta\left(x-x_{j}\right) \frac{\partial}{\partial x_{j}}- \\
-\frac{\pi \beta}{2 l} \sum_{j \neq k=\overline{1, N}}\left(\delta\left(x-x_{j}\right) \operatorname{ctg}\left[\frac{\pi}{l}\left(x_{j}-x_{k}\right)\right]+\delta\left(x-x_{k}\right) \operatorname{ctg}\left[\frac{\pi}{l}\left(x_{k}-x_{j}\right)\right]\right)
\end{gathered}
$$

in the Hilbert space $L_{2}^{(s)}\left([0, l]^{N} ; \mathbb{C}\right), N \in \mathbb{Z}_{+}$, parametrized by a running point $x \in \mathbb{R} /[0, l] \mathbb{Z}$. The corresponding secondly quantized representation of the operator (90) looks as

$$
\mathrm{D}(x)=\psi^{+}(x) \psi_{x}(x)-\frac{\pi \beta}{l} \int_{0}^{l} d y \operatorname{ctg}\left[\frac{\pi}{l}(x-y)\right]: \psi^{+}(x) \psi^{+}(y) \psi(y) \psi(x):
$$

for any $x \in \mathbb{R} /[0, l] \mathbb{Z}$, or in the density operator $\rho: \Phi \rightarrow \Phi$ form as

$$
\begin{gathered}
\mathrm{D}(x)=\psi^{+}(x) \psi_{x}(x)- \\
-\frac{\pi \beta}{2 l} \int_{0}^{l} d y\left[\operatorname{ctg}\left[\frac{\pi}{l}(x-y)\right]: \rho(x) \rho(y):-\operatorname{ctg}\left[\frac{\pi}{l}(y-x)\right]: \rho(y) \rho(x):\right] .
\end{gathered}
$$

Now, based on the operator (92), one can formulate the following proposition.

Proposition 1. The secondly quantized Hamiltonian operator (89) in the Fock space $\Phi$ is representable in dual to (85) factorized form as

$$
\mathrm{H}=\int_{0}^{l} d x \mathrm{D}^{+}(x) \rho(x)^{-1} \mathrm{D}(x)+\mathrm{E}
$$

where the ground state energy operator $\mathrm{E}: \Phi \rightarrow \Phi$ equals

$$
\mathrm{E}=\frac{1}{3}\left(\frac{\pi \beta}{l}\right)^{2}: \mathrm{N}^{3}:+\left(\frac{\pi \beta}{l}\right)^{2}: \mathrm{N}^{2}:
$$

where

$$
\mathrm{N}:=\int_{0}^{l} \rho(x) d x
$$

is the particle number operator, and satisfies the determining conditions

$$
(\mathrm{H}-\mathrm{E}) \mid \Omega)=0, \mathrm{D}(x) \mid \Omega)=0
$$

on the suitably renormalized vacuum ground state $\mid \Omega) \in \Phi$ for all $x \in \mathbb{R} /[0, l] \mathbb{Z}$. Moreover, for any integer $N \in \mathbb{Z}_{+}$the corresponding projected vector $\left.\left.\mid \Omega_{N}\right):=\mid \Omega\right)\left.\right|_{\Phi_{N}}$ there hold satisfies the following eigenfunction relationships:

$$
\begin{aligned}
\left.\mathrm{N} \mid \Omega_{N}\right) & \left.\left.\left.=N \mid \Omega_{N}\right), \quad \mathrm{E} \mid \Omega_{N}\right)=\left(\frac{1}{3}\left(\frac{\pi \beta}{l}\right)^{2}: \mathrm{N}^{3}:+\left(\frac{\pi \beta}{l}\right)^{2}: \mathrm{N}^{2}:\right) \mid \Omega_{N}\right)= \\
& \left.=\left[\frac{1}{3}\left(\frac{\pi \beta}{l}\right)^{2}\left(N^{3}-3 N^{2}+2 N\right)+\left(\frac{\pi \beta}{l}\right)^{2} N(N-1)\right] \mid \Omega_{N}\right)= \\
& \left.=\left[\frac{1}{3}\left(\frac{\pi \beta}{l}\right)^{2}\left(N^{3}-3 N^{2}+2 N+3 N^{2}-3 N\right)\right] \mid \Omega_{N}\right)= \\
& \left.\left.=\left[\frac{1}{3}\left(\frac{\pi \beta}{l}\right)^{2} N\left(N^{2}-1\right)\right] \mid \Omega_{N}\right):=E_{N} \mid \Omega_{N}\right),
\end{aligned}
$$

coinciding exactly with the result (86). 
Remark 5. When deriving the expression (97), we have used the identity

$$
\begin{gathered}
\rho(x) \rho(y)=: \rho(x) \rho(y):+\rho(y) \delta(x-y) \\
\rho(x) \rho(y) \rho(z)=: \rho(x) \rho(y) \rho(z):+: \rho(x) \rho(y): \delta(y-z)+ \\
+: \rho(y) \rho(z): \delta(z-x)+: \rho(z) \rho(x): \delta(x-y)+: \rho(x) \delta(y-z) \delta(z-x),
\end{gathered}
$$

which holds $[1,3,6,20]$ for the density operator $\rho: \Phi \rightarrow \Phi$ at any points $x, y, z \in \mathbb{R} /[0, l] \mathbb{Z}$.

Observe now that the operator (91) can be rewritten down as

$$
\mathrm{D}(x)=\mathrm{K}(x)-\mathrm{A}(x),
$$

where, by definition,

$$
\mathrm{K}(x):=\psi^{+}(x) \psi_{x}(x), \mathrm{A}(x):=\frac{\pi \beta}{l} \int_{0}^{l} d y \operatorname{ctg}\left[\frac{\pi}{l}(x-y)\right]: \rho(x) \rho(y):
$$

for all $x \in \mathbb{R} /[0, l] \mathbb{Z}$. Recalling now the second condition of (96), one can rewrite it equivalently as

$$
\mathrm{K}(x) \mid \Omega)=\mathrm{A}(x) \mid \Omega)
$$

on the renormalized ground state vector $\mid \Omega) \in \Phi$ for all $x \in \mathbb{R} /[0, l] \mathbb{Z}$. On the other hand, owing to the expression (93), we obtain the searched for current algebra representation

$$
\mathrm{H}=\int_{0}^{l} d x\left(\mathrm{~K}^{+}(x)-\mathrm{A}(x)\right) \rho(x)^{-1}(\mathrm{~K}(x)-\mathrm{A}(x))
$$

of the Calogero-Sutherland Hamiltonian operator (83) in the suitably renormalized Fock space $\Phi$, as it was already demonstrated in the work [20,27], using the condition (101) in the form (39).

Discuss now shortly the quantum integrability of the Calogero-Sutherland model (83). Owing to the factorized representation (102) one can easily observe that for any integer $p \in \mathbb{Z}_{+}$the operators $\mathrm{h}(x):=\mathrm{D}^{+}(x) \rho(x)^{-1} \mathrm{D}(x): \Phi \rightarrow \Phi, x \in \mathbb{R} /[0, l] \mathbb{Z}$, commute to each other and with the particle number operator $\mathrm{N}: \Phi \rightarrow \Phi$, that is

$$
[\mathrm{h}(x), \mathrm{h}(y)]=0,[\mathrm{~h}(x), \mathrm{N}]=0
$$

for any $x, y \in \mathbb{R} /[0, l] \mathbb{Z}$. As a result of the commutation property (103) one easily obtains that for any integer $p \in \mathbb{Z}_{+}$the symmetric operators

$$
\mathrm{H}^{(p)}:=\int_{0}^{l} d x \mathrm{~h}(x)^{p}
$$

also commute to each other

$$
\left[\mathrm{H}^{(p)}, \mathrm{H}^{(q)}\right]=0
$$

for all integers $p, q \in \mathbb{Z}_{+}$, and in particular, commute to the Calogero-Sutherland Hamiltonian operator (93):

$$
\left[\mathrm{H}^{(p)}, \mathrm{H}\right]=0 .
$$

Concerning the related $N$-particle differential expressions for the operators (104), it is enough to calculate their projections on the $N$-particle Fock subspace $\Phi_{N} \subset \Phi, N \in \mathbb{Z}_{+}$. Namely, let an arbitrary vector $\left.\mid \varphi_{N}\right) \in \Phi_{N}$ is representable as

$$
\left.\left.\mid \varphi_{N}\right):=\int_{[0, l]^{N}} f_{N}\left(x_{1}, x_{2}, \ldots, x_{N}\right) \prod_{j=1, N} d x_{j} \psi^{+}\left(x_{j}\right) \mid 0\right)
$$


for some coefficient function $f_{N} \in L_{2}^{(s)}\left([0, l]^{N} ; \mathbb{C}\right)$. Then, by definition,

$$
\left.\left.\mathrm{H}^{(p)} \mid \varphi_{N}\right):=\mid \varphi_{N}^{(p)}\right),
$$

where

$$
\left.\left.\mid \varphi_{N}^{(p)}\right)=\int_{[0, l]^{N}}\left(H_{N}^{(p)} f_{N}\right)\left(x_{1}, x_{2}, \ldots, x_{N}\right) \prod_{j=\overline{1, N}} d x_{j} \psi^{+}\left(x_{j}\right) \mid 0\right)
$$

for a given $p \in \mathbb{Z}_{+}$any any $N \in \mathbb{Z}_{+}$. In particular, for $p=2$, when $\mathrm{H}^{(2)}+\mathrm{E}=\mathrm{H}: \Phi \rightarrow \Phi$, one easily retrieves the shifted Calogero-Sutherland Hamiltonian operator (83):

$$
H_{N}^{(2)}=-\sum_{j=\overline{1, N}} \frac{\partial^{2}}{\partial x_{j}^{2}}+\sum_{j \neq k=1, N} \frac{\pi^{2} \beta(\beta-1)}{l^{2} \sin ^{2}\left[\frac{\pi}{l}\left(x_{j}-x_{k}\right)\right]}-\left(\frac{\pi \beta}{l}\right)^{2} \frac{N\left(N^{2}-1\right)}{3} .
$$

Respectively for higher integers $p>2$ the resulting $N$-particle differential operator expressions $H_{N}^{(p)}: L_{2}^{(s)}\left([0, l]^{N} ; \mathbb{C}\right) \rightarrow L_{2}^{(s)}\left([0, l]^{N} ; \mathbb{C}\right), N \in \mathbb{Z}_{+}$, can be obtained the described above way by means of simple yet well cumbersome calculations, and which will prove to be completely equivalent to those, calculated before in the cited before nice work [26].

Remark 6. In the thermodynamical limit, when $\lim _{N \rightarrow \infty, l \rightarrow \infty} N / \pi l:=\bar{\rho}>0$, the structural operator $\mathrm{D}(x): \Phi \rightarrow \Phi, x \in \mathbb{R} /[0, l] \mathbb{Z}$, reduces to

$$
\overline{\mathrm{D}}(x):=\lim _{N / l \rightarrow \bar{\rho}} \mathrm{D}(x)=\psi^{+}(x) \psi_{x}(x)-\beta \int_{\mathbb{R}} d y \frac{: \rho(y) \rho(x):}{x-y},
$$

and respectively, the operator (83) reduces to

$$
\bar{H}_{N}=-\sum_{j=1, N} \frac{\partial^{2}}{\partial x_{j}^{2}}+\beta(\beta-1) \sum_{j \neq k=1, N} \frac{1}{\left(x_{j}-x_{k}\right)^{2}}
$$

in the Hilbert space $L_{2}^{(s)}\left(\mathbb{R}^{N} ; \mathbb{C}\right)$ for any $N \in \mathbb{Z}_{+}$, whose secondly quantized operator expression in the suitable Fock space $\Phi$ equals

$$
\overline{\mathrm{H}}=\int_{\mathbb{R}} d x\left(\overline{\mathrm{D}}^{+}(x) \rho(x)^{-1} \overline{\mathrm{D}}(x)+\epsilon_{0}\right),
$$

where $\epsilon_{0}:=\lim _{N / l \rightarrow \bar{\rho}} \frac{E_{N}}{l}=\bar{\rho}^{3} / 3$ denotes the average energy density of the reduced Calogero-Sutherland Hamiltonian operator (112) as $N \rightarrow \infty$, exactly coinciding with the before obtained results in [20].

\section{Conclusions}

In the work we succeeded in developing an effective algebraic scheme of constructing density operator and functional representations for the canonical local quantum current algebra and its application to quantum Hamiltonian and symmetry operators reconstruction. We analyzed the corresponding factorization structure for quantum Hamiltonian operators, governing spatially manyand one-dimensional integrable dynamical systems. The quantum generalized oscillatory and Calogero-Sutherland models of spin-less bose-particles were analyzed in detail.

Author Contributions: The problem posing and a factorization concept were suggested by A.P.; the operator structure analysis, current algebra representations and calculations of examples were done by D.P.

Funding: This research received no external funding.

Acknowledgments: Authors would like to convey their warm thanks to Gerald Goldin, Joel Lebowitz, Denis Blackmore, Nikolai N. Bogolubov (Jr.), Maciej Błaszak and Anatol Odziewicz for instructive discussions, useful comments and remarks. A special A.P.'s appreciation belongs to Joel Lebowitz for the invitation to take part in the 121-st Statistical Mechanics Conference, held 12-14 May 2019 in the Rutgers University, New Brunswick, 
NJ, USA. His acknowledgement also belongs to the Department of Physics, Mathematics and Computer Science of the Cracov University of Technology for a local research grant F-2/370/2018/DS.

Conflicts of Interest: The authors declare no conflict of interest.

\section{References}

1. Berezin, F.A. The Method of Second Quantization; Academic Press: Cambridge, MA, USA, 1966.

2. Berezin, F.A.; Shubin, M.A. Schrödinger Equation; Springer: London, UK, 2012; 555p.

3. Bogolubov, N.N.; Bogolubov, N.N., Jr. Introduction into Quantum Statistical Mechanics; World Scientific: Hackensack, NJ, USA, 1986.

4. Blackmore, D.; Prykarpatsky, A.K.; Samoylenko, V.H. Nonlinear Dynamical Systems of Mathematical Physics: Spectral and Differential-Geometrical Integrability Analysis; World Scientific Publ.: Hackensack, NJ, USA, 2011.

5. Faddeev, L.D.; Yakubovskii, O.A. Lectures on Quantum Mechanics for Mathematics Students; American Mathematical Society: Providence, RI, USA, 2009.

6. Goldin, G.A.; Grodnik, J.; Powers, R.T.; Sharp, D. Nonrelativistic current algebra in the N/V limit. J. Math. Phys. 1974, 15, 88-100. [CrossRef]

7. Prykarpatsky, A.; Mykytyuk, I. Algebraic Integrability of Nonlinear Dynamical Systems on Manifolds: Classical and Quantum Aspects; Kluwer Academic Publishers: Dordrecht, The Netherlands, 1998.

8. Takhtajan, L.A. Quantum Mechanics for Mathematicians; Department of Mathematics, Stony Brook University: Stony Brook, NY, USA, 2008.

9. Berezanskii, Y.M. Expansions in Eigenfunctions of Selfadjoint Operators; Translations of Mathematical Monographs; AMS: Ann Arbor, MI, USA, 1968; 809p.

10. Berezansky, Y.M.; Kondratiev, Y.G. Spectral Methods in Infinite Dimensional Analysis; v.1 and 2, Kluwer; Springer: London, UK, 1995.

11. Dirac, P.A.M. The Principles of Quantum Mechanics, 2nd ed.; Clarendon Press: Oxford, UK, 1935.

12. Fock, V.A. Konfigurationsraum und zweite Quantelung. Z. Phys. 1932, 75, 622-647. [CrossRef]

13. Prykarpatsky, A.K.; Taneri, U.; Bogolubov, N.N., Jr. Quantum Field Theory and Application to Quantum Nonlinear Optics; World Scientific: New York, NY, USA, 2002.

14. Bogolubov, N.N., Jr.; Prykarpatsky, A.K. Quantum method of generating Bogolubov functionals in statistical physics: Current Lie algebras, their representations and functional equations. Phys. Elem. Part. At. Nucl. 1986, 17, 791-827.

15. Goldin, G.A. Nonrelativistic current algebras as unitary representations of groups. J. Math. Phys. 1971, 12, 462-487. [CrossRef]

16. Goldin, G.A.; Menikoff, R.; Sharp, F.H. Diffeomorphism groups, gauge groups, and quantum theory. Phys. Rev. Lett. 1983, 51, 2246-2249. [CrossRef]

17. Goldin, G.A.; Menikoff, R.; Sharp, F.H. Representations of a local current algebra in nonsimply connected space and the Aharonov-Bohm effect. J. Math. Phys. 1981, 22, 1664-1668. [CrossRef]

18. Aref'eva, I.Y. Current formalism in nonrelativistic quantum mechanics. Theor. Math. Phys. 1972, 10, $146-155$. [CrossRef]

19. Feenberg, E. Ground state of an interacting boson system. Ann. Phys. 1974, 84, 128-137. [CrossRef]

20. Menikoff, R.; Sharp, D. Representation of a local current algebra: Their dynamical determination. J. Math. Phys. 1975, 16, 2341-2352. [CrossRef]

21. Albeverio, S.; Daletsky, A.; Kondratiev, Y.; Lytvynov, E. Laplace operators in de-Rham complexes associated with measures on configuration spaces. J. Geom. Phys. 2003, 47, 259-302. [CrossRef]

22. Berezansky, Y.M. A generalization of white noice analysis by means of theory of hypergroups. Rep. Math. Phys. 1996, 38, 289-300. [CrossRef]

23. Prykarpatsky, A.; Bogolubov, N., Jr.; Golenia, J.; Taneri, U. Introductive Backgrounds to Modern Quantum Mathematics with Application to Nonlinear Dynamical Systems. Int. J. Theor. Phys. 2008, 47, 2882-2897. [CrossRef]

24. Araki, H. Hamiltonian Formalism and the Canonical Commutation Relations in Quantum Field Theory. J. Math. Phys. 1960, 1, 492-504. [CrossRef]

25. Pardee, W.J.; Schlessinger, L. Wright. J. Phys Rev. 1968, 165, 1883. 
26. Lapointe, L.; Vinet, L. Exact operator solution of the Calogero-Sutherland model. Commun. Math. Phys. 1996, 178, 425-152. [CrossRef]

27. Menikoff, R. Generating functionals determining representation of a nonrelativistic local current algebra in the N/V limit. J. Math. Phys. 1974, 15, 1394-1408. [CrossRef]

28. Dunkl, C.F. Differential-difference operators associated to reflection groups. Trans. Am. Math. Soc. 1989, 311, 167-183. [CrossRef]

(C) 2019 by the authors. Licensee MDPI, Basel, Switzerland. This article is an open access article distributed under the terms and conditions of the Creative Commons Attribution (CC BY) license (http://creativecommons.org/licenses/by/4.0/). 